EPITHELIAL CELL BIOLOGY

\title{
Key role of the sympathetic microenvironment for the interplay of tumour necrosis factor and interleukin 6 in normal but not in inflamed mouse colon mucosa
}

\author{
R H Straub, K Stebner, P Härle, F Kees, W Falk, J Schölmerich
}

See end of article for authors' affiliations

Correspondence to: Professor R H Straub, Laboratory of Neuro/ Endocrino/Immunology, Department of Internal Medicine I, University Hospital Regensburg, 93042 Regensburg, Germany; rainer.straub@ klinik.uni-regensburg.de

Revised version received 25 February 2005

Accepted for publication 4 April 2005

Published online first 21 April 2005
Background: In the intestinal tract, the role of sympathetic neurotransmitters has been largely ignored in mucosal neuroimmunology.

Aim: Our aim was to investigate the influence of the sympathetic microenvironment on the mucosal interplay of tumour necrosis factor (TNF) and interleukin 6 (IL-6).

Methods: Colon strips of normal and colitic BALB/c mice were superfused in vitro. Tissue was electrically stimulated to investigate the influence of endogenous norepinephrine (NE) on secretion of IL-6, with or without anti-TNF antibodies (anti-TNF) and adrenoceptor antagonists. IL-6 was secreted from macrophages.

Results: Superfusion with anti-TNF stimulated IL-6 secretion in normal but not in colitic colon $(p<0.005)$. Parallel superfusion with a $\beta$-adrenergic antagonist abrogated this phenomenon. Anti-TNF increased release of NE from normal colonic strips $(p<0.05)$, which demonstrates TNF induced inhibition of preterminal NE release. In colitic mice, anti-TNF did not change NE release. In the presence of anti-TNF, exogenous and endogenous NE stimulated colonic IL-6 secretion via $\beta$-adrenoceptors in normal $(p<0.001)$ but not in colitic mice. In the absence of anti-TNF, endogenous and exogenous NE inhibited IL-6 secretion via the $\beta$-adrenoceptor in normal but not in colitic mice $(p<0.01)$. Colitic mice demonstrated loss of sympathetic nerve fibres.

Conclusions: Modulation of mucosal IL-6 is largely dependent on the sympathetic microenvironment and availability of local TNF in normal but not in colitic mice. Anti-TNF strategies may lead to an increase in the proinflammatory cytokine depending on adrenergic tone. This would be relevant with normal sympathetic innervation, which is lost in colitic mice. We present a model of sympathetic regulation of colonic macrophage TNF and IL-6 secretion. l: is generally acknowledged that the sympathetic nervous system of the intestinal tract regulates intramural blood flow, epithelial cell proliferation, intestinal fluid transport, mucous glycoprotein secretion, and intestinal motility. ${ }^{1-7}$ However, its direct immunomodulating properties have partly been ignored although norepinephrine (NE) via $\beta$-adrenoceptors ( $\beta A R)$ is a strong inhibitor of, for example, tumour necrosis factor (TNF) secretion, $^{8-10}$ interferon $\gamma$ stimulated MHC class II expression, ${ }^{11}{ }^{12}$ phagocytosis, ${ }^{13}{ }^{14}$ and many other immune functions. The sympathetic nerve fibre density is extraordinarily high in the intestinal wall due to a very dense vasculature. ${ }^{15}$ Initial studies on sympathoimmune interplay demonstrated that intestinal inflammation suppressed the release of NE from the myenteric plexus. ${ }^{16}$ In addition, cytokines such as interleukin (IL)-1 $\beta$, IL-6, and TNF inhibited release of NE from intestinal nerve fibres. ${ }^{17-19}$ This should have an impact on adrenergic neurotransmission during inflammatory episodes leading to increased gastrointestinal motility. ${ }^{20}$ The same group demonstrated that NE stimulates IL-1 $\beta$ induced IL- 6 secretion from isolated intestinal smooth muscle cells via $\beta A R .^{21}$ One group demonstrated that chemical sympathectomy reduced the number of ovalbumin specific immune cells but increased the number of IgA positive and $\operatorname{IgM}$ positive cells in the intestinal lamina propria. $^{22}{ }^{23}$ However, direct interplay between sympathetic nerve fibres and immune cells has not been studied because techniques were not available which would permit direct investigation of the influence of endogenously released neurotransmitters on intestinal immune cell function in isolated intact compartments of intestinal tissue.
Using a superfusion technique, we were able to characterise sympathetic nerve-immune cell interplay in spleen slices (reviewed by Straub ${ }^{24}$ ). Electrical field stimulation induced release of endogenous neurotransmitters. ${ }^{25}$ Studies with specific antagonists revealed that the endogenous sympathetic neurotransmitters NE, endogenous opioids, and neuropeptide Y modulate IL-6 and TNF secretion of splenic macrophages. ${ }^{26}$ These studies demonstrated that IL-6 is the downstream readout parameter of adrenergic TNF regulation when TNF is switched on during bacterial infection. ${ }^{26}$ In this situation, IL- 6 downstream of TNF is inhibited via $\beta A R$. In contrast, if TNF secretion is negligible, adrenergic regulation of IL-6 is reversed and its secretion is stimulated via $\beta$ AR. ${ }^{26}$ This demonstrates the intricate interrelation of NE and these two cytokines under immune stimulated and unstimulated conditions.

This present study aimed to investigate the major source of IL-6 in the intestinal wall. In addition, we aimed to study the neuroimmune interplay of endogenous NE, TNF, and IL-6 using a superfusion technique with colon strips from normal and colitic BALB/c mice. We focused on $\beta A R$ mediated effects of NE for this interplay between TNF and IL-6. Furthermore, we examined the influence of TNF on neuronal NE release

Abbreviations: $\beta A R, \beta$-adrenoceptors; anti-TNF, neutralising anti-TNF antibodies; DSS, dextran sodium sulphate; ELISA, enzyme linked immunosorbent assay; HPLC, high performance liquid chromatography; IFN- $\gamma$, interferon $\gamma$; IL, interleukin; NE, norepinephrine; PBS, phosphate buffered saline; SAP, saporin; TBS, Tris buffered saline; TNF, tumour necrosis factor 
and studied sympathetic innervation of the colon in normal and colitic mice. In our discussion we link the results of this study to anti-TNF therapy in patients.

\section{MATERIALS AND METHODS}

\section{Animals}

A total of 100 female BALB/C mice (8-10 weeks; 26-34 g; Charles River, Sulzfeld, Germany) were used. The exact numbers of animals used are given in the figure legends. Animals were fed a standard laboratory chow diet. All procedures were in accordance with the guidelines for the care and use of laboratory animals approved by the University of Regensburg Institutional Animal Care and Use Committee (Government of Oberpfalz AZ 621-2531.1-22/02).

\section{Animal model of chronic colitis}

The model of chronic colitis has been described previously. ${ }^{27}$ Dextran sodium sulphate (DSS, molecular weight 40 000; ICN, Inc., Eschwege, Germany) chronic colitis was induced by feeding $2 \%$ DSS over four cycles. In parallel, mice received a standard laboratory chow diet. One cycle consisted of feeding $2 \%$ DSS in drinking water for seven days followed by a period of 10 days drinking water without DSS. Thirty days after the last cycle, animals were killed and the colon was removed for further investigation (superfusion technique, see below). Of the $100 \mathrm{BALB} / \mathrm{c}$ used, a total of 25 were included in animal experiments with chronic colitis (Government of Oberpfalz AZ 621-2531.1-22/02).

\section{Immunohistochemical localisation of IL-6 secreting cells and macrophages in the mucosa}

Colon strips were transferred to superfusion chambers and superfused for two hours with $10 \mu \mathrm{g} / \mathrm{ml}$ Brefeldin A (SigmaAldrich, Deisenhofen, Germany) in order to prevent cytokine excretion (superfusion technique, see below). Strips were removed from the superfusion chambers and embedded in Tissue-Tek (Tissue-Tek, Sakura Finetek Europe, Zoeterwoude, the Netherlands) and snap frozen. Tissue samples were cut into $5 \mu \mathrm{m}$ thick sections. Several pairs of consecutive cryosections were placed on different precoated slides (SuperFrost Plus; Menzel-Gläser, Braunschweig, Germany) and placed in a solution with $0.5 \% \mathrm{H}_{2} \mathrm{O}_{2}$ in $0.05 \mathrm{M}$ Tris buffered saline (TBS) in order to destroy endogenous peroxidases. Slides were then washed $3 \times$ 10 minutes in $0.05 \mathrm{M}$ TBS.

For IL-6 immunohistochemistry, sections were blocked for one hour with a solution of $10 \%$ whole rabbit serum (Dako, Hamburg, Germany) in 0.05 M TBS containing $1 \%$ bovine serum albumin and $0.01 \% \mathrm{NaN}_{3}$ (Sigma, Deisenhofen, Germany). Slides were then washed $2 \times$ five minutes in $0.05 \mathrm{M}$ TBS. Tissue sections were incubated in a humid chamber for 12-18 hours with purified polyclonal rabbit antibodies against IL-6 (dilution 1:250; Acris via DPC Biermann, Bad Nauheim, Germany). After another $3 \times$ five minutes washes with $0.05 \mathrm{M}$ TBS, secondary biotinylated goat antirabbit antibodies were used (Dako). This complex was visualised using the Vectastain ABC kit (Vector Lab., Burlingame, California, USA) and the AEC substrate kit for peroxidase (Vector Lab.), which produced a red reaction product. Slides were counterstained with haematoxylin. Under control conditions, the primary antibodies were not used in the above mentioned protocol.

For macrophage staining, we used a primary monoclonal rat antimouse antibody against the Mac-3 antigen (dilution 1:1000, clone M3/84; BD Bioscience Pharmingen, Heidelberg, Germany). An Alexa 546 conjugated secondary goat antirat antibody (Molecular Probes, Leiden, the Netherlands) was used to achieve immunofluorescent staining of macrophages.
Under control conditions, the respective isotype was used in the above mentioned protocol (dilution 1:1000).

\section{Elimination of intestinal macrophages}

Anti-Mac-1 (CDllb) antibodies conjugated to the ribosome inactivating protein saporin (Mac-1 IgG SAP) were used to eliminate intestinal macrophages, as previously described. ${ }^{28}$ Mice were treated on day -3 and day -1 before sacrifice with $20 \mu \mathrm{g}$ of Mac-1 IgG SAP, SAP alone, anti-Mac-1 without SAP, and rat IgG conjugated to SAP (rat IgG SAP). At least three animals for each condition were used. On day 0 , colon strips were transferred to superfusion chambers and superfused for five hours to study spontaneous IL-6 secretion as a possible readout parameter of macrophages (cytokine determination and superfusion technique, see below). In a further experiment, mice were preloaded from day -5 to day -1 with $250 \mu \mathrm{g}$ rat IgG per day to block unspecific binding of rat IgG SAP to Fcy receptors (rat IgG from Sigma).

\section{Cytokine determination}

As TNF was not measurable in superfusate (not shown; superfusion technique, see below), we used the downstream parameter IL-6 which is much more stable and was secreted in large amounts. Murine IL-6 in superfusate fractions was determined by sandwich enzyme linked immunosorbent assays (ELISAs; Endogen via Perbio Science, Bonn, Germany). Detection limit was $<3 \mathrm{pg} / \mathrm{ml}$, and intra-assay and interassay coefficients of variation were below $10 \%$.

\section{Superfusion medium, preparation of the tissue, superfusion protocol, and standardisation of the tissue}

The colon (including the ascending, transverse, and half of the descending colon) of the above mentioned BALB/c mice was carefully removed after cervical dislocation and opening of the peritoneal cavity. The colon was opened using scissors to cut the intestinal wall in a longitudinal direction. The opened colon was washed several times in ice cold culture medium (RPMI 1640, 25 mM HEPES, 5\% fetal calf serum, $30 \mu \mathrm{M}$ mercaptoethanol, $0.57 \mathrm{mM}$ ascorbic acid, $1.3 \mathrm{mM}$ calcium, $100 \mathrm{U} / \mathrm{ml}$ penicillin, and $100 \mu \mathrm{g} / \mathrm{ml}$ streptomycin (all from Sigma), and $8 \mu \mathrm{g} / \mathrm{ml}$ ciprofloxacin (Bayer, Leverkusen, Germany)). After washing, small strips of the entire colonic wall with a size of approximately $25 \mathrm{~mm}^{2}$ were cut and randomly transferred to superfusion chambers. These chambers had a volume of $80 \mu \mathrm{l}$ and were equipped with platinum electrodes forming the bottom and top of each chamber, respectively. ${ }^{25}$ We used colon strips from different parts of the colon which were randomly transferred to the superfusion chambers in a blinded manner.

The superfusion method has been described previously. ${ }^{25}$ Briefly, superfusion was performed for 5.5 hours at a temperature of $37^{\circ} \mathrm{C}$ and a flow rate of $66 \mu \mathrm{l} / \mathrm{min}$ (one strip per chamber, $4 \times 8=32$ chambers in parallel; one mouse with 32 strips per day). During the first 2.5 hours of the superfusion period, all strips were superfused with culture medium without any additional drugs or electrical stimulation. Between 120 and 150 minutes, superfusate was collected to determine IL- $6_{3 \mathrm{~h}}$ (ELISA technique, see above). During the second part of the superfusion period (3rd-5th hour), drug, electrical stimulation, or both, were applied to modulate IL-6 secretion. Between the 300th and 330th minute, superfusate was collected a second time to determine IL- $6_{5 h}$. IL- 6 at three hours was used to standardise the IL- 6 secreting capacity of the different strips. The dimensionless ratio $\psi=100 \times\left(\mathrm{IL}-6_{5 \mathrm{~h}} /\right.$ IL- $6_{3 h}$ ) was used to standardise IL-6 secretion of each strip at 5.5 hours. ${ }^{26}$ In experiments with spleen slices, this standardisation technique was found to be superior to standardisation using the leucocyte count of the slice, wet weight, dry weight, 
A

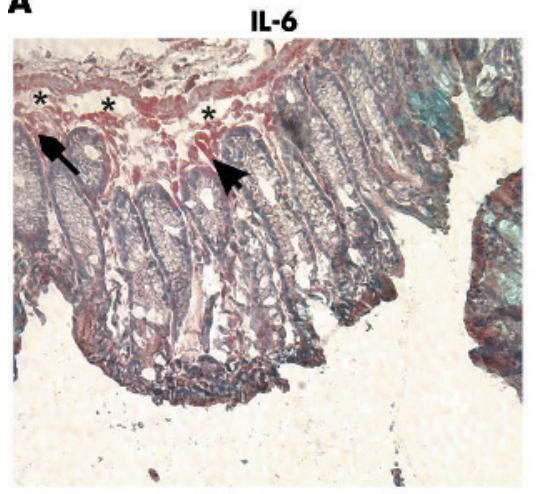

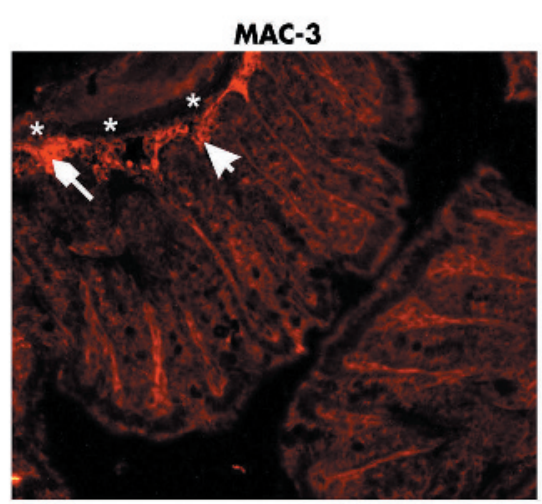

B

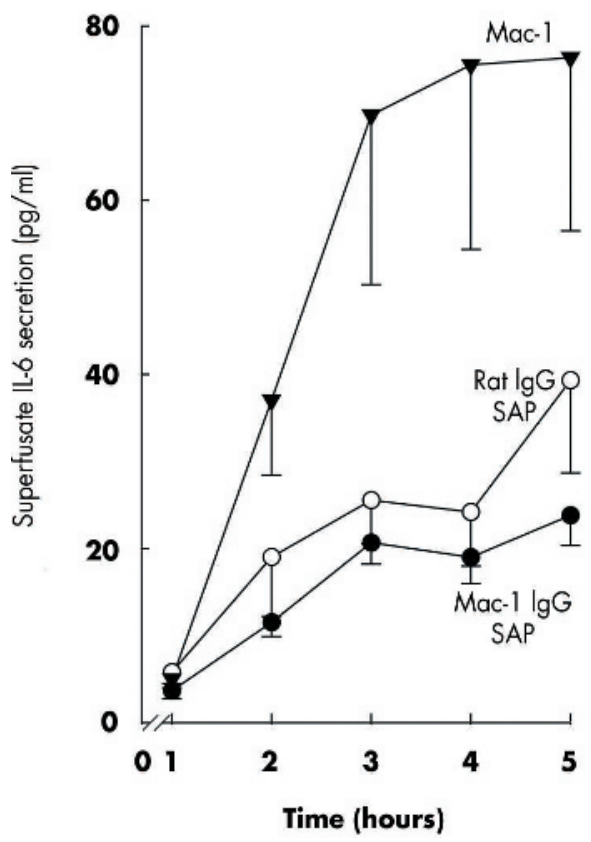

With rat $\lg G$ preloading

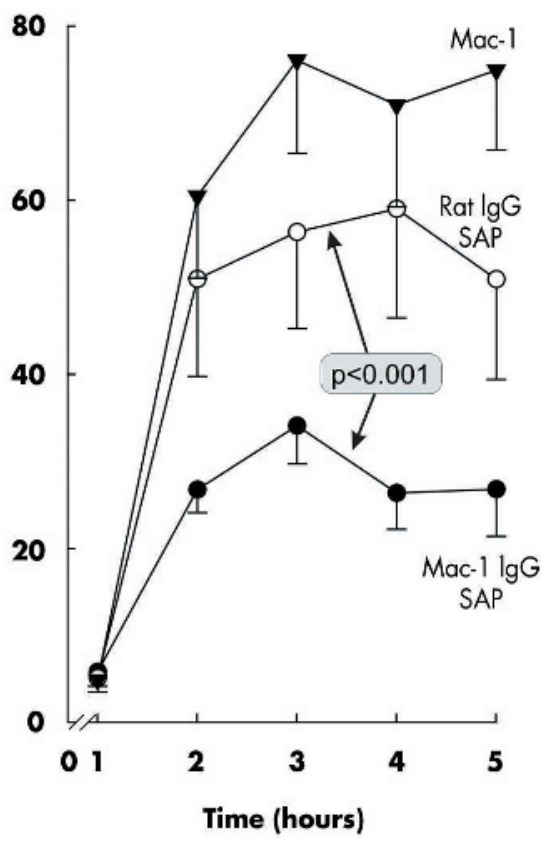

Figure 1 Allocation of cellular interleukin 6 (IL-6) to cells in the intestinal wall. (A) Sequential staining of cryosections for IL-6 (red cells, left panel) and Mac-3 (fluorescent cells, right panel). Asterisks and arrows demonstrate similar microscopic structures in both high power fields. Cell nuclei are stained with haematoxylin. Magnification $400 \times$. (B) Secretion of IL-6 from colonic wall strips after treatment with Mac-1 specific antibodies conjugated to the ribosome inactivating protein saporin (Mac-1 lgG-SAP), a control rat lgG conjugated to saporin (rat lgG SAP), and an antibody to Mac-1 without saporin (similar to control superfusion without addition of any substances). The left panel demonstrates experiments in at least three mice (per condition, $n \geqslant 9$ mice) without rat lgG preloading, whereas the right panel shows experiments in at least three mice (per condition, $n \geqslant 9$ mice) with rat lgG preloading $(250 \mu \mathrm{g} / \mathrm{mouse})$ over five days prior to sacrifice. The $p$ value is for comparison of Mac-1 lgG-SAP and rat $\lg G$ SAP. or the volume of the slice. ${ }^{26}$ Tissue viability of colon strips was excellent after 5.5 hours, as estimated by NE release, histology, and lactate dehydrogenase (LDH) release.

\section{Experiments with adrenergic agonists and adrenergic} antagonists, and electrical stimulation

To study the effects of adrenergic agonists, NE (Sigma) and isoproterenol ( $(\beta 1 / \beta 2$ adrenergic agonist; Sigma) were used. In all experiments the substances were added between the 170th and the 330th minute of superfusion without additional electrical stimulation.

To indirectly study the effect of electrically released endogenous $N E,^{29}{ }^{30}$ nadolol $(\beta 1 / \beta 2$ adrenergic receptor antagonist; Sigma) was used at the concentrations indicated. Dilutions of the drugs were prepared immediately before the experiments. In experiments to study transmitter effects, drugs were added at 150 minutes until the end of superfusion. After a drug equilibration period of 20 minutes between the 150th and the 170th minute, slices were electrically stimulated using one train of monophasic rectangular pulses ( $2 \mathrm{~ms}, 5 \mathrm{~Hz}, 43 \mathrm{~mA}, 45000$ pulses) which lasted until the end of superfusion.
Experiments with the monoclonal neutralising antiTNF antibody VlqH8

As it is known that secretion of TNF precedes that of IL-6, the significance of TNF for IL-6 secretion was characterised using the monoclonal anti-TNF antibody VlqH8. ${ }^{31}$ In these experiments, purified VlqH8 was used at the concentrations indicated, between the 150th and the 330th minute of superfusion. Control experiments were performed with a respective IgG.

\section{Determination of superfusate norepinephrine}

NE was determined by high performance liquid chromatography (HPLC) with electrochemical detection. NE was isolated from superfusate using a commercially available kit for the determination of catecholamines in plasma (Recipe, Munich, Germany). The HPLC system consisted of a pump model K-1001 (Knauer, Berlin, Germany), an autosampler model SIL-9A (Shimadzu, Duisburg, Germany), an analytical column (length $\times$ inner diameter $150 \times 4.6 \mathrm{~mm}$ ) filled with Luna C18(2) $5 \mu \mathrm{m}$ silicagel (Phenomenex, Aschaffenburg, Germany), an electrochemical detector model L-3500A (Recipe), and the class 10 integration software (Shimadzu, Duisburg, Germany). Analytical column and detector cell 

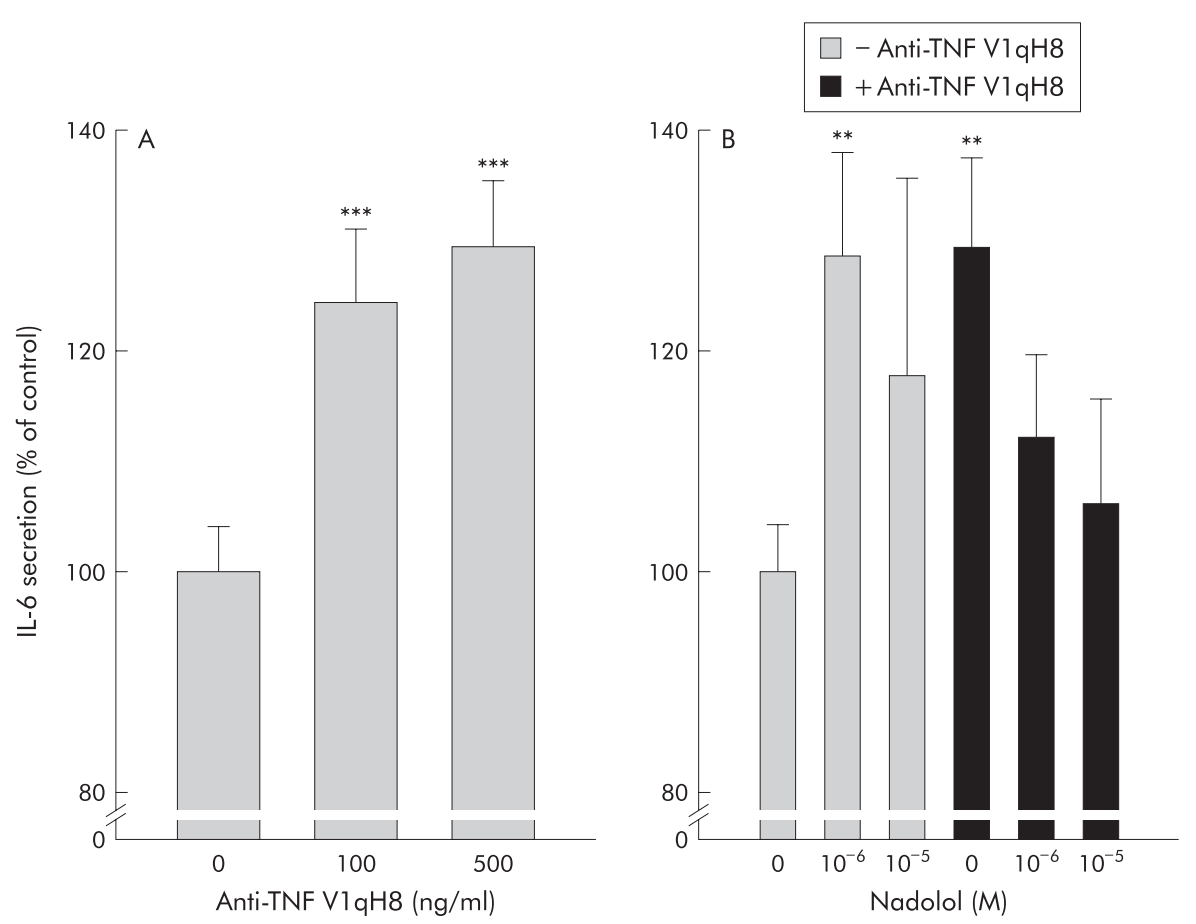

Figure 2 Role of tumour necrosis factor (TNF) for colonic interleukin 6 (IL-6) secretion and influence of endogenous $\beta$-adrenoceptor ( $\beta A R$ ) pathways $(A, C)$. Influence of anti-TNF antibodies V1 qH8 on spontaneous IL-6 secretion from colonic strips in normal mice (A) and colitic mice (C). At least 24 colon strips were used in normal and colitic mice $(n \geqslant 6$ mice). ( $B, D)$ Influence of the $\beta A R$ antagonist nadolol on spontaneous IL-6 secretion in the absence and presence of anti-TNF $\mathrm{VlqH} 8$ in normal (B) and colitic (D) mice. At least 24 colon strips were used in normal and colitic mice ( $n \geqslant 6$ mice). ${ }^{* *} p<0.01$, ${ }^{* * *} p<0.005$ compared with controls (zero).
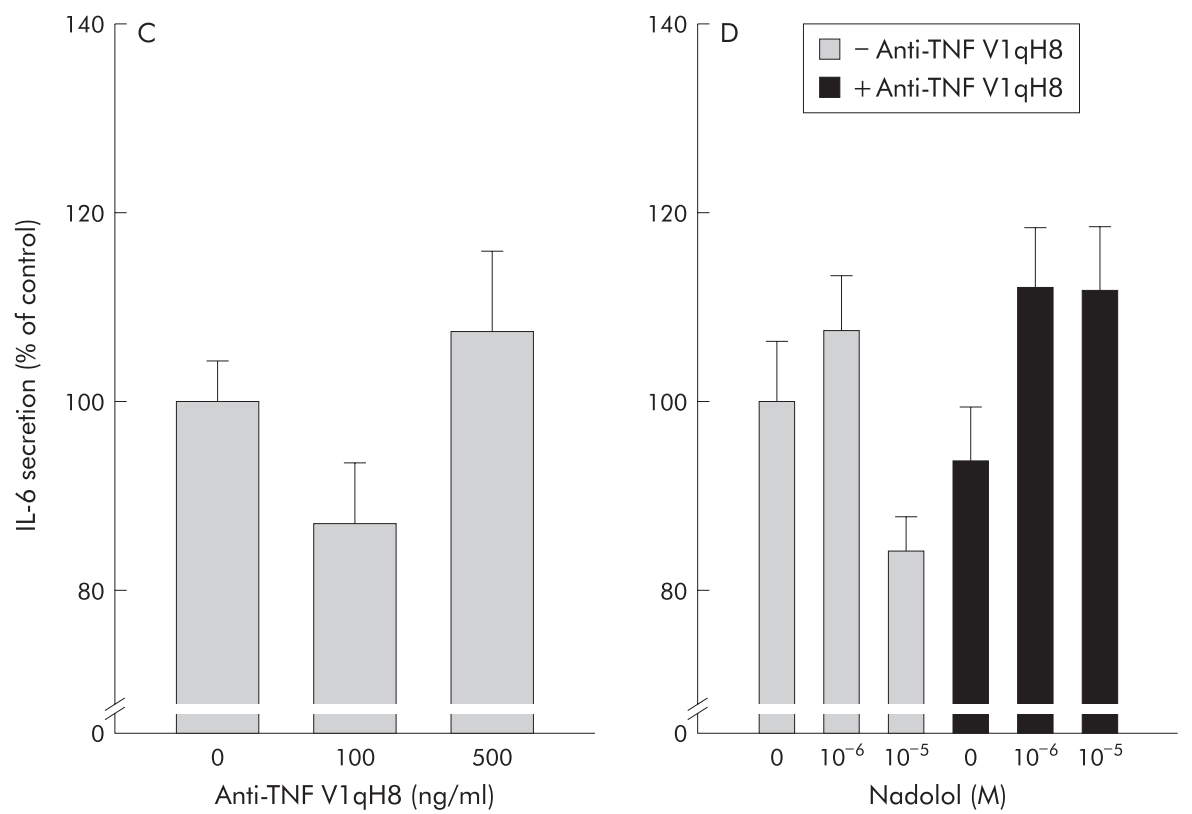

(model sputnik with glassy carbon working electrode set at $0.50 \mathrm{~V} v \mathrm{Ag} / \mathrm{AgCl}$; Recipe) were mounted in a high pressure liquid chromatography thermostat HT3000 (Recipe) maintained at $25^{\circ} \mathrm{C}$. The mobile phase was mixed with $6.90 \mathrm{~g}$ $\mathrm{NaH}_{2} \quad \mathrm{PO}_{4} \times \mathrm{H}_{2} \mathrm{O}, \quad 1.80 \mathrm{~g} \mathrm{NaOH}, 100 \mathrm{mg}$ sodium octane sulphonate, $50 \mathrm{mg} \mathrm{Na}{ }_{2}$ EDTA, $3.0 \mathrm{ml}$ acetic acid, $1000 \mathrm{ml}$ water, $40 \mathrm{ml}$ acetonitrile, and $20 \mathrm{ml}$ methanol (resulting $\mathrm{pH}$ 5.4). At a flow rate of $1.0 \mathrm{ml} / \mathrm{min}, \mathrm{NE}$ eluted after 4.5 minutes and the internal standard dihydroxy benzylamine after 7.9 minutes. Using $2.5 \mathrm{ml}$ of perfusate, the limit of quantitation was $5 \mathrm{pg} / \mathrm{ml}$ for NE.

\section{Immunohistochemistry of sympathetic nerve fibres in the colon}

Colon pieces from normal and colitic animals were washed and immediately fixed after sacrifice in $4 \%$ formalin for

12 hours. Tissue was then washed in phosphate buffered saline (PBS) and incubated in $20 \%$ sucrose in PBS for another 12 hours. Thereafter, tissue was embedded in protective freezing medium (Tissue-Tek; Sakura Finetek Europe, Zoeterwoude, the Netherlands) and quick frozen floating on liquid nitrogen for nerve fibre staining or embedded in paraffin for standard haematoxylin-eosin staining. Cryosections were immunostained with primary antibodies against tyrosine hydroxylase (Chemicon, Temecula, California, USA), the key enzyme for NE production in sympathetic nerve endings, according to a protocol described previously. ${ }^{32}$ An Alexa 546 conjugated secondary goat antirat antibody (Molecular Probes, Leiden, the Netherlands) was used to achieve immunofluorescent staining. Under control conditions, the respective isotype was used in the above mentioned protocol. 

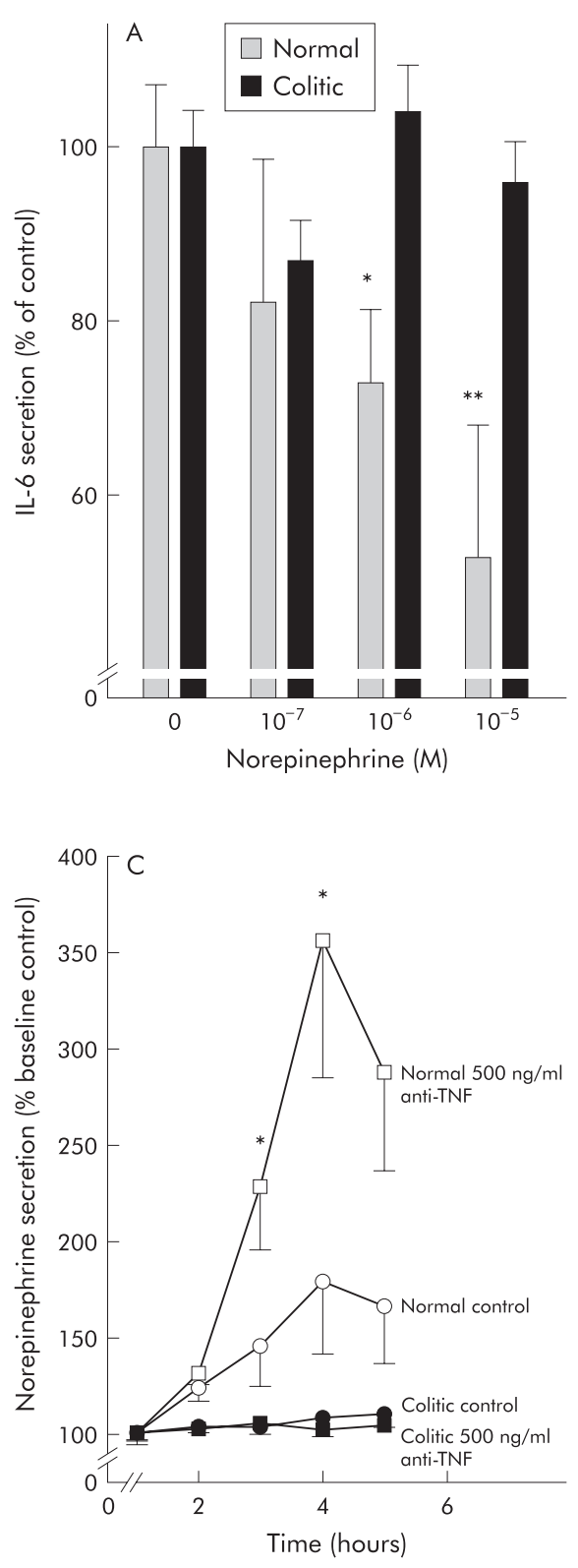

\section{Statistical analysis}

All data are given as mean (SEM). In order to compare entire curves over time, the general linear model procedure was used (SPSS for Windows V1 1.5.1; SPSS Inc., Chicago, Illinois, USA). The unpaired $t$ test (SPSS) was used to compare group means. A p value of $<0.05$ was considered significant.

\section{RESULTS}

Allocation of cellular IL- 6 to cells in the intestinal wall In order to visualise IL- 6 secreting cells, a pair of consecutive cryosections were immunohistochemically stained for either IL-6 or Mac-3. IL-6 immunohistochemistry stained large cells in and close to the muscularis mucosa and also between crypts (fig 1A). In the consecutive cryosection, Mac-3 positive cells matched to the area of IL-6 staining (compare fig 1B with fig $1 \mathrm{~A}$ ).

To further investigate the role of macrophages for IL- 6 secretion, experiments with antibodies conjugated to the ribosome inactivating protein SAP were carried out. In experiments without rat IgG preloading, administration of anti-Mac-1 IgG-SAP, or rat IgG-SAP demonstrated marked inhibition of IL-6 secretion in relation to administration of anti-Mac-1 antibodies alone (similar to the control curve without any substance; not shown, fig lB left panel). This indicates that administration of saporin conjugated antibodies eliminates approximately $66 \%$ of IL-6 secreting cells. However, the effect seemed to be unspecific because rat IgGSAP, which does not specifically bind to macrophages, also showed a similar effect (fig lB left panel). In order to avoid the unspecific effect, mice were pretreated with a high dose of rat IgG intraperitoneally for five days prior to sacrifice. Preloading with rat IgG avoided the unspecific effect with rat IgG-SAP (fig $1 \mathrm{~B}$ right panel). Both experiments indicate that a major portion of IL-6 is secreted from macrophages.

\section{Role of TNF for colonic IL-6 secretion and influence of endogenous $\beta A R$ pathways}

Experiments with anti-TNF antibodies demonstrated an increase in IL-6 secretion from colonic strips (fig 2A). This 

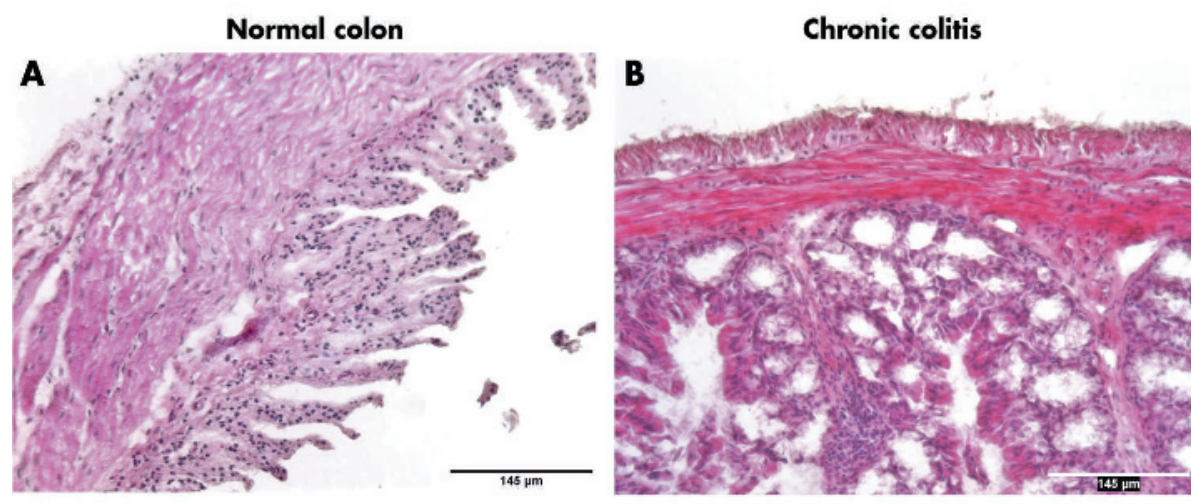

Figure 4 Sympathetic innervation of the colon in normal $(A, C)$ and colitic $(B$, D) mice. (A, B) Haematoxylin-eosin staining of colonic tissue. (C, D) In subsequent cross sections, sympathetic nerve fibres were stained by antibodies against tyrosine hydroxylase and secondary fluorescence labelled antibodies. White arrowheads indicate bundles of sympathetic nerve fibres in normal colon. All micrographs were taken at $100 \times$ magnification.
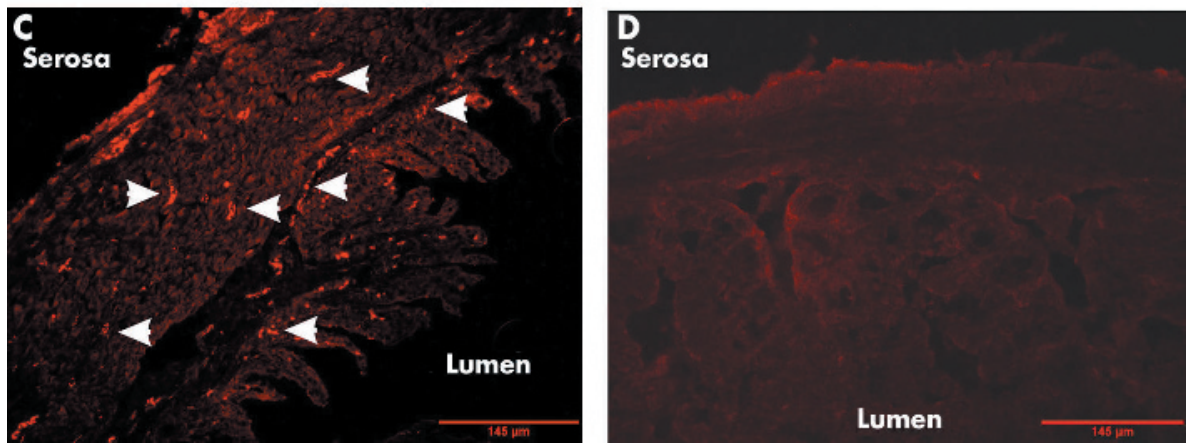

was surprising because TNF normally stimulates downstream IL-6 secretion and thus we expected a decrease in IL-6 secretion, as demonstrated previously in murine spleen slices. ${ }^{26}$ In animal with chronic colitis, anti-TNF antibodies did not modulate spontaneous IL-6 secretion (fig 2C). In order to study the influence of the $\beta$ AR pathway, the $\beta A R$ antagonist nadolol was used. Nadolol completely inhibited the increase in colonic IL- 6 secretion in the presence of antiTNF (fig 2B). This indicates that the increase in IL-6 secretion in the absence of TNF is mediated by endogenous NE via $\beta A R$. However, in the absence of anti-TNF, nadolol increased IL-6 secretion (fig 2B), which indicates that under these conditions IL- 6 is inhibited via $\beta A R$ pathways. These effects were not observed in animals with chronic colitis (fig 2D).
To corroborate these findings, the influence of NE on colonic IL-6 secretion was investigated. NE dose dependently inhibited colonic IL-6 secretion in the absence of anti-TNF (fig 3A), which was not observed in chronic colitic mice (fig 3A). As NE at high concentrations (it then binds with high affinity to $\beta \mathrm{AR}$ ) demonstrated the strongest inhibition of IL-6 secretion under these conditions, we assumed a role for $\beta A R$. Indeed, in the presence of anti-TNF, the $\beta A R$ agonist isoproterenol dose dependently increased IL-6 secretion (fig 3B). In chronic colitic animals, this effect was markedly reduced (fig 3B). This indicates a switch in the influence of $\beta A R$ in the absence and presence of TNF in normal but not in colitic mice. Furthermore, these experiments also suggested an inhibitory influence of TNF on NE secretion.

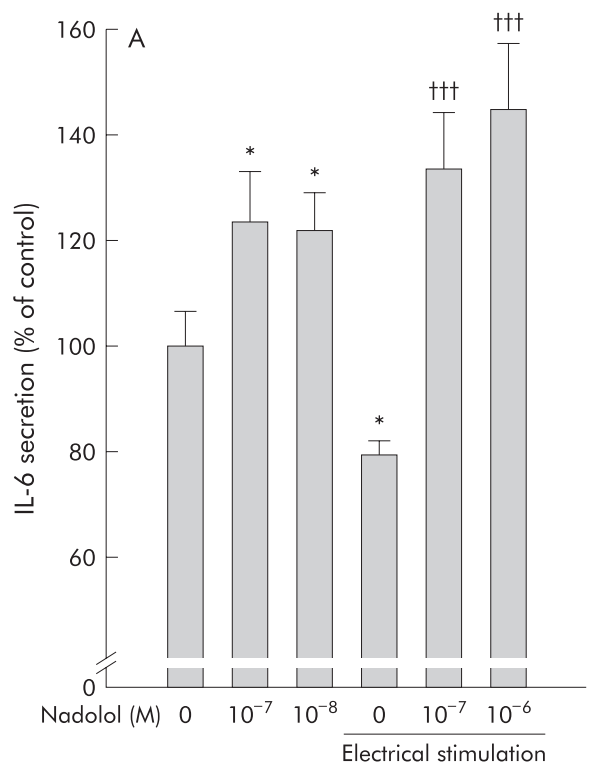

Figure 5 Role of tumour necrosis factor (TNF) for colonic interleukin 6 (IL-6) secretion and influence of endogenous $\beta$-adrenoceptor ( $\beta A R$ ) pathways. (A) Influence of the $\beta A R$ antagonist nadolol on spontaneous IL-6 secretion in unstimulated and electrically stimulated colon strips. At least 32 colon strips were used per condition ( $n \geqslant 8$ mice). ${ }^{*} p<0.05$ compared with controls (zero without electrical stimulation); $\uparrow+\dagger p<0.001$ compared with control electrical stimulation (zero with electrical stimulation). (B) Influence of anti-TNF, nadolol, and electrical stimulation on IL-6 secretion. At least 24 colon strips were used per condition $(n \geqslant 12)$. 


\section{NE secretion under the influence of TNF and electrical stimulation}

Superfusion experiments in the presence of anti-TNF demonstrated a dose dependent increase in NE secretion from colonic strips in normal mice (fig 3C) but not in colitic mice (fig 3C), which indicates that TNF inhibits preterminal NE release in normal mice. The constant increase in NE under control conditions most likely represents spontaneous $\mathrm{NE}$ release from nerve terminals or NE producing cells (fig 3C).

In the above experiments, we demonstrated the influence of exogenous $\beta$-adrenergic agonists and antagonists. In order to study the influence of endogenously released NE, colon strips were electrically stimulated in order to release NE. NE was secreted on electrical stimulation in normal mice only and not in colitic mice (fig 3D). Interestingly, the level of NE secretion was somewhat increased in colitic mice but electrical stimulation did not change the outflow (fig 3D).

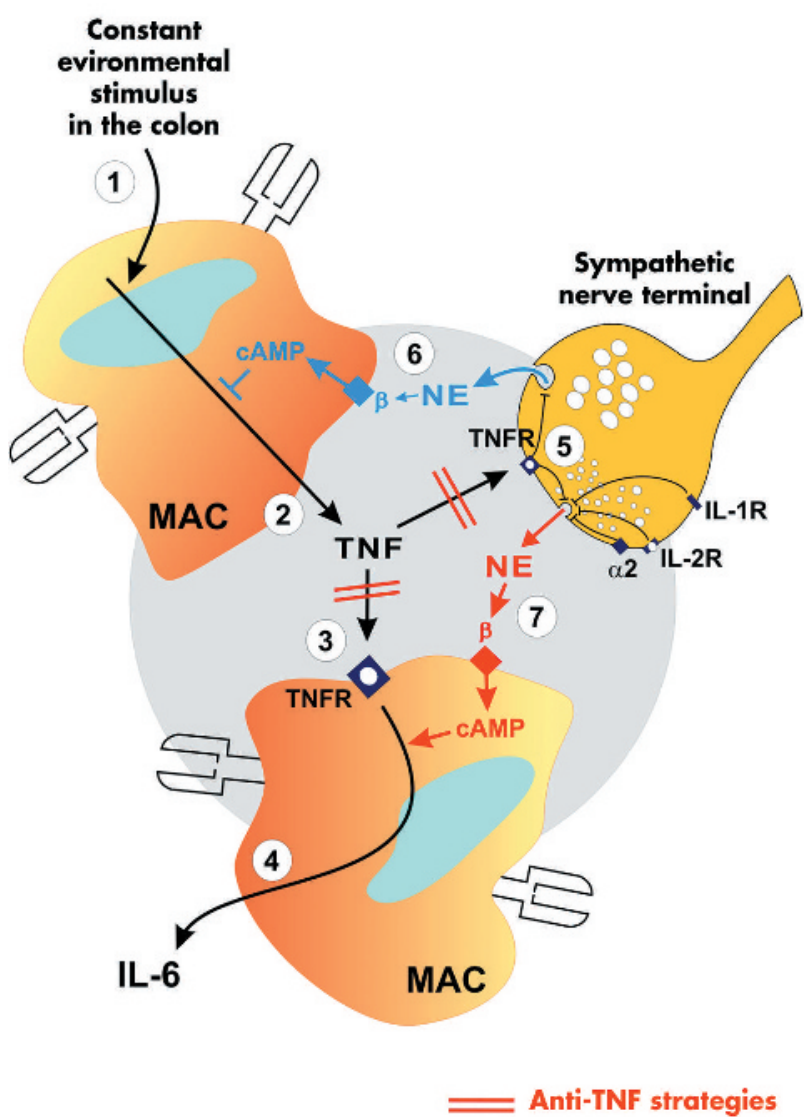

Figure 6 Model of colonic interleukin 6 (IL-6) regulation by tumour necrosis factor (TNF) and norepinephrine (NE) in normal colon. A line with an arrow (a bar) indicates a stimulatory (inhibitory) pathway. Constant environmental stimuli provoke local TNF secretion from, for example, macrophages (MAC (1)). (2) TNF is secreted into the local microenvironment and binds to TNF receptors (TNFR) on macrophages (3) and sympathetic nerve terminals (5). TNF stimulates downstream IL-6 secretion which becomes the readout parameter of TNF regulation (4). Under conditions without anti-TNF, the blue $\beta$-adrenoceptor ( $\beta A R$ ) pathway (6) dominates the red $\beta A R$ pathway (7), which leads to NE induced inhibition of TNF and subsequently IL- 6 secretion. In the presence of anti-TNF, the influence of TNF is completely blocked and the effects mediated by the blue $\beta A R$ pathway are negligible. Under these conditions, the red $\beta A R$ pathway dominates and IL- 6 is stimulated via an increase in extracellular NE and intracellular cAMP. Other cytokines such as IL-1 or IL-2 inhibit NE release from sympathetic nerve terminals.

\section{Sympathetic innervation of the colon in normal and} colitic mice

As the findings were largely different in normal compared with chronic colitic mice, we investigated sympathetic innervation of the colon. Figure 4 is representative of three normal and three colitic mice. Sympathetic innervation was lost in colitic animals.

Role of TNF for colonic IL-6 secretion and influence of endogenous NE via $\beta A R$ in normal mice

As sympathetic nerve fibres were absent in colitic animals, the following experiments with electrical stimulation to release endogenous NE were only conducted in normal mice. The experiments above in the absence of anti-TNF demonstrated inhibition of IL- 6 secretion via $\beta A R$ in normal mice. In experiments with electrical stimulation, IL-6 secretion was significantly inhibited (fig 5A, fourth column from the left). This electrically induced inhibition of IL- 6 was blunted in the presence of nadolol (fig 5A, columns 5 and 6 from the left), which corroborates experiments with NE (fig 3A). Nadolol itself also stimulated IL-6 secretion (fig 5A, columns 2 and 3). The inhibiting effect of electrical stimulation was abrogated in the presence of anti-TNF (fig 5B, column $3 v$ column 4 ) but restored by concomitant superfusion with anti-TNF plus nadolol (fig 5B, column $4 v 5$ ). Again, these experiments demonstrate a clear switch in the influence of endogenous NE in the presence and absence of anti-TNF.

\section{DISCUSSION}

This study in the colon of mice demonstrates the opposing $\beta A R$ effects of endogenous NE on IL-6 secretion in the absence and presence of TNF in normal but not in colitic mice. Sympathetic regulation of TNF is contrary to regulation of IL-6. NE via $\beta A R$ inhibits TNF secretion by increasing intracellular cyclic $\mathrm{AMP}^{8-10}$ but NE via cyclic AMP stimulates IL-6 secretion..$^{21}{ }^{33-36}$ Thus in the presence of TNF, NE inhibits TNF secretion and downstream IL-6 secretion via $\beta$ ARs, but in the absence of TNF, IL- 6 is directly stimulated via $\beta$ ARs (similar to the infected spleen, see Straub and colleagues ${ }^{26}$ ). This model of sequential cytokine regulation in normal mice is detailed in fig 6. It is obvious that NE can have very different effects depending on the presence or absence of TNF.

Conditions in the colon are quite different from conditions in the spleen (spleen data reviewed by Straub ${ }^{24}$ ). In the colon, continuous microenvironmental stimuli such as bacteria or other stimuli constantly stimulate TNF secretion. This is different from the spleen because the role of TNF on downstream IL-6 secretion is much smaller under sterile conditions in the spleen. ${ }^{26}$ As demonstrated here in normal colon, and in earlier studies, ${ }^{19}$ elevated TNF in the colon inhibits sympathetic NE release which probably leads to upregulation of $\beta A R$ pathways under normal conditions (sensitisation). After TNF neutralisation, increased release of endogenous NE now stimulates IL-6 secretion via the sensitised $\beta A R$. This only happens in normal but not in colitic mice because the latter animals have lost the sympathetic innervation in the inflamed tissue. Loss of sympathetic innervation is not colitis specific because it has also been demonstrated in rheumatoid arthritis ${ }^{32}$ and insulitis in diabetic rats. ${ }^{37}$ In the spleen of NMRI and BALB/c mice, TNF neutralisation inhibits IL-6 secretion which is completely independent of $\beta A R .{ }^{26}$ Thus in the spleen, sensitisation of the $\beta A R$ pathway does not exist.

From our point of view, this has an important implication for TNF neutralising strategies because anti-TNF would lead to an increase in IL-6 in the colon but to inhibition of IL-6 in the spleen. Thus anti-TNF strategies can have largely different effects depending on the micromilieu in a certain 
tissue. Immune stimuli together with the sympathetic microenvironment can thus alter the influence of cytokine neutralising strategies. We do not know if this alteration leads to an unfavourable outcome in patients with colonic inflammation, such as ulcerative colitis. Several uncontrolled studies have indicated that anti-TNF strategies are not impressively effective in ulcerative colitis. ${ }^{38}$ Whether or not the presently ongoing studies ACT1 and ACT2 will demonstrate favourable results is not known at present. Nevertheless, one may speculate that in areas of normal colon in patients with ulcerative colitis, anti-TNF antibodies may stimulate IL-6 secretion, which can be unfavourable. In contrast, in areas of inflammation, anti-TNF antibodies would not influence IL-6 secretion (via NE), which is surprising because TNF is a strong stimulator of IL-6 secretion.

In conclusion, this study indicates that modulation of mucosal IL-6 is largely dependent on the local context of sympathetic innervation and the presence of TNF in normal but not in colitic colon (which depends on loss of sympathetic innervation in colitic mice). TNF is an important proinflammatory cytokine in many different inflammatory cascades. TNF stimulates downstream proinflammatory events such as release of cytokines (for example, IL-6) and matrix metalloproteinases which degrade the tissue, and TNF can stimulate expression of adhesion molecules and chemotaxis of different infiltrating leucocytes, such as neutrophils and others. As such, TNF is a key element for proinflammatory pathways in the intestinal mucosa. This proinflammatory cascade is thought to be inhibited by anti-TNF strategies. However, anti-TNF strategies may not always inhibit proinflammatory pathways. This study demonstrates that antiTNF strategies may lead to an increase in concentration of the proinflammatory cytokine IL-6 in the normal colon. Thus anti-TNF strategies may even cause a proinflammatory situation which depends on local noradrenergic tone. Depending on the balance of pro- and anti-inflammatory effects, anti-TNF strategies can be favourable or unfavourable in a given disease. This study provides clues as to whether or not, under certain circumstances, anti-TNF strategies can even elicit a proinflammatory milieu. The reactions described herein largely depend on the micromilieu in a specific tissue. Similar contextual phenomena probably occur with other neutralising drugs. This study may help in understanding tissue specific modulation of cytokine secretion which depends on neuronal innervation.

\section{ACKNOWLEDGEMENTS}

This work was supported by the DFG (Deutsche Forschungsgemeinschaft: Sonderforschungsbereich $585, \mathrm{~B} 8$ ) and by the institution. We thank Nicole Steigerwald and Melanie Grünbeck for excellent technical assistance.

\section{Authors' affiliations}

R H Straub, K Stebner, P Härle, W Falk, J Schölmerich, Department of Internal Medicine I, University Hospital Regensburg, Regensburg, Germany

F Kees, Department of Pharmacology, University of Regensburg, Germany

Conflict of interest: None declared.

\section{REFERENCES}

1 Gershon MD. Inhibition of gastrointestinal movement by sympathetic nerve stimulation: the site of action. J Physiol 1967;189:317-27.

2 Hulten L, Lindhagen J, Lundgren $\mathrm{O}$. Sympathetic nervous control of intramural blood flow in the feline and human intestines. Gastroenterology 1977;72:41-8

3 Tutton PJ, Barkla DH. The influence of adrenoceptor activity on cell proliferation in colonic crypt ipithelium and in colonic adenocarcinomata. Virchows Arch B Cell Pathol 1977;24:139-46.
4 Sjovall H, Redfors S, Hallback DA, et al. The effect of splanchnic nerve stimulation on blood flow distribution, villous tissue osmolality and fluid and electrolyte transport in the small intestine of the cat. Acta Physiol Scand 1983:117:359-65.

5 Shepherd AP, Riedel GL. Intramural distribution of intestinal blood flow during sympathetic stimulation. Am J Physiol 1988;255:H1091-5.

6 Mullol J, Rieves RD, Baraniuk JN, et al. The effects of neuropeptides on mucous glycoprotein secretion from human nasal mucosa in vitro. Neuropeptides 1992;21:231-8.

7 Vanner S, Surprenant A. Neural reflexes controlling intestinal microcirculation. Am J Physiol 1996;271:G223-30.

8 Renz H, Gong JH, Schmidt A, et al. Release of tumor necrosis factor-alpha from macrophages. Enhancement and suppression are dose-dependently regulated by prostaglandin E2 and cyclic nucleotides. J Immunol 1988;141:2388-93.

9 Severn A, Rapson NT, Hunter CA, et al. Regulation of tumor necrosis factor production by adrenaline and beta-adrenergic agonists. J Immunol 1992; 148:3441-5

10 van der Poll T, Jansen J, Endert E, et al. Noradrenaline inhibits lipopolysaccharide-induced tumor necrosis factor and interleukin 6 production in human whole blood. Infect Immun 1994;62:2046-50.

11 Frohman EM, Vayuvegula B, van den Noort S, et al. Norepinephrine inhibits gamma-interferon-induced MHC class II (la) antigen expression on cultured brain astrocytes. J Neuroimmunol 1988;17:89-101.

12 Loughlin AJ, Woodroofe MN, Cuzner ML. Modulation of interferon-gammainduced major histocompatibility complex class II and Fc receptor expression on isolated microglia by transforming growth factor-beta 1, interleukin-4, noradrenaline and glucocorticoids. Immunology 1993;79:125-30.

13 Abrass CK, O'Connor SW, Scarpace PJ, et al. Characterization of the betaadrenergic receptor of the rat peritoneal macrophage. J Immunol 1985; 135: 1338-41.

14 Mitra S, Ghosh L, Chakrabarty P, et al. Effect of bioamines on uptake of promastigotes of Leishmania donovani by hamster peritoneal macrophages. $J$ Med Microbiol 1992;36:283-7.

15 Furness JB, Costa $M$. Types of nerves in the enteric nervous system. Neuroscience 1980:5:1-20.

16 Swain MG, Blennerhassett PA, Collins SM. Impaired sympathetic nerve function in the inflamed rat intestine. Gastroenterology 1991;100:675-82.

17 Hurst S, Collins SM. Interleukin-1 beta modulation of norepinephrine release from rat myenteric nerves. Am J Physiol 1993;264:G30-5.

18 Ruhl A, Hurst S, Collins SM. Synergism between interleukins 1 beta and 6 on noradrenergic nerves in rat myenteric plexus. Gastroenterology 1994; 1077:993-1001.

19 Hurst SM, Collins SM. Mechanism underlying tumor necrosis factor-alpha suppression of norepinephrine release from rat myenteric plexus. Am J Physiol 1994;266:G1123-9.

20 Xia Y, Hu HZ, Liu S, et al. IL-1 beta and IL- 6 excite neurons and suppress nicotinic and noradrenergic neurotransmission in guinea pig enteric nervous system. J Clin Invest 1999;103:1309-16.

21 Van Assche G, Barbara G, Deng Y, et al. Neurotransmitters modulate cytokine-stimulated interleukin 6 secretion in rat intestinal smooth muscle cells. Gastroenterology 1999;116:346-53.

22 Gonzalez-Ariki S, Husband AJ. Ontogeny of $\lg A(+)$ cells in lamina propria: effects of sympathectomy. Dev Comp Immunol 2000;24:61-9.

23 Gonzalez-Ariki S, Husband AJ. The role of sympathetic innervation of the gut in regulating mucosal immune responses. Brain Behav Immun 1998; 12:53-63.

24 Straub RH. Complexity of the bi-directional neuroimmune junction in the spleen. Trends Pharmacol Sci 2004;25:640-6.

25 Straub RH, Lang B, Falk W, et al. In vitro superfusion method for the investigation of nerve-immune cell interaction in murine spleen. J Neuroimmunol 1995;61:53-60.

26 Straub RH, Linde HJ, Männel DN, et al. A bacteria-induced switch of sympathetic effector mechanisms augments local inhibition of TNF-alpha and IL-6 secretion in the spleen. FASEB J 2000;14:1380-8.

27 Kojouharoff G, Hans W, Obermeier F, et al. Neutralization of tumour necrosis factor (TNF) but not of IL-1 reduces inflammation in chronic dextran sulphate sodium-induced colitis in mice. Clin Exp Immunol 1997; 107:353-8.

28 Kanai T, Watanabe M, Okazawa A, et al. Macrophage-derived IL-18mediated intestinal inflammation in the murine model of Crohn's disease. Gastroenterology 2001;121:875-88.

29 Straub RH, Herrmann M, Frauenholz T, et al. Neuroimmune control of interleukin- 6 secretion in the murine spleen. Differential-adrenergic effects of electrically released endogenous norepinephrine under various endotoxin conditions. J Neuroimmunol 1996:71:37-43.

30 Straub RH, Herrmann M, Berkmiller G, et al. Neuronal regulation of interleukin 6 secretion in murine spleen: Adrenergic and opioidergic control J Neurochem 1997;68:1633-9.

31 Echtenacher B, Falk W, Männel DN, et al. Requirement of endogenous tumor necrosis factor/cachectin for recovery from experimental peritonitis. J Immunol 1990; 145:3762-6

32 Miller LE, Jüsten HP, Schölmerich J, et al. The loss of sympathetic nerve fibers in the synovial tissue of patients with rheumatoid arthritis is accompanied by increased norepinephrine release from synovial macrophages. FASEB J 2000; 14:2097-107.

33 Norris JG, Benveniste EN. Interleukin-6 production by astrocytes: Induction by the neurotransmitter norepinephrine. J Neuroimmunol 1993;45:137-45. 
34 Maimone D Cioni $C$, Rosa $S$, et al. Norepinephrine and vasoactive intestinal peptide induce IL-6 secretion by astrocytes: Synergism with IL-1 beta and TNFalpha. J Neuroimmunol 1993;47:73-82.

35 Huang QH, Takaki A, Arimura A. Central noradrenergic system modulates plasma interleukin-6 production by peripheral interleukin-1. Am J Physiol 1997;273:R731-8.

36 Szabo C, Hasko G, Zingarelli B, et al. Isoproterenol regulates tumour necrosis factor, interleukin-10, interleukin-6 and nitric oxide production and protects against the development of vascular hyporeactivity in endotoxaemia. Immunology 1997;90: 95-100.

37 Mei Q, Mundinger TO, Lernmark A, et al. Early, selective, and marked loss of sympathetic nerves from the islets of BioBreeder diabetic rats. Diabetes 2002;51:2997-3002

38 Schölmerich J, Huber G. Biological therapy in IBD. Anti-tumor necrosis factoralpha and others. Dig Dis 2003;21:180-91.

\section{EDITOR'S QUIZ: GI SNAPSHOT}

\section{An unusual case of biliary pain}

\section{Clinical presentation}

A 62 year old woman presented with a one month history of increasing postprandial epigastric pain radiating through to her back. Her medical history included endoscopic sphincterotomy of the papilla with laparoscopic cholecystectomy two years earlier for biliary lithiasis. Her clinical evaluation was normal. White cell count was $14600 / \mathrm{mm}^{3}$, and liver aminotransferases, serum lipase, and amylase were all normal. A stress test and echocardiography were normal. No abnormalities were found at gastroscopy, on abdominal ultrasound, or after tomography. An endoscopic ultrasound was performed (fig 1). What does it show?

\section{Question}

What is the differential diagnosis?

See page 1145 for answer

This case is submitted by:

J Boujaoude

Department of Gastroenterology, Hotel-Dieu de France Hospital, SaintJoseph University, Beirut, Lebanon

B Abboud

Department of General Surgery, Hotel-Dieu de France Hospital, Saint-Joseph University, Beirut, Lebanon

M Chemaly

Department of Gastroenterology, Hotel-Dieu de France Hospital, SaintJoseph University, Beirut, Lebanon

K Hachem

Department of Radiology, Hotel-Dieu de France Hospital, Saint-Joseph University, Beirut, Lebanon

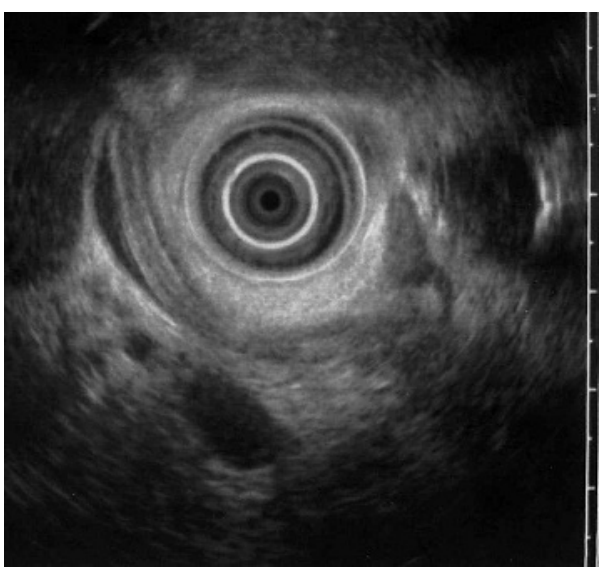

Figure 1 Endoscopic ultrasound.

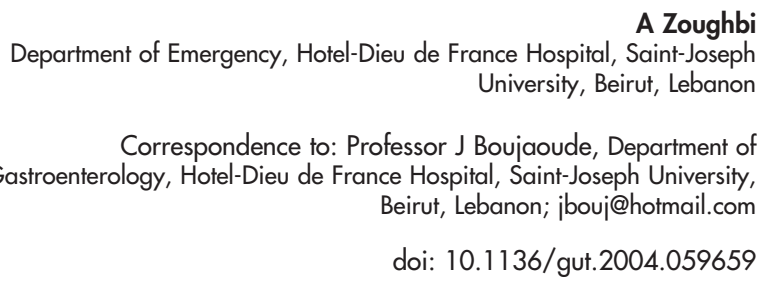

